\title{
Localization Analysis of Nonlocal Model Based ON CRACK InTERACTIONS
}

\author{
By Milan Jirásek ${ }^{1}$ and Zdeněk P. Bažant, ${ }^{2}$ Fellow, ASCE
}

\begin{abstract}
Abstaact: The conventional nonlocal model, often used as a localization limiter for continuum-based constitutive laws with strain-softening, has been based on an isotropic averaging function. It has recently been shown that this type of nonlocal averaging leads to a model that cannot satisfactorily reproduce experimental results for very different test geometries without modifying the value of the characteristic length depending on geometry. A micromechanically based enrichment of the nonlocal operator by a term taking into account the directional dependence of crack interactions can be expected to improve the performance of the nonlocal model. The aim of this paper is to examine this new model in the context of a simple localization problem reducible to a one-dimensional description. Strain localization in an infinite layer under plane stress is studied using both the old and the new nonlocal formulations. The importance of a renormalization of the averaging function in the proximity of a boundary is demonstrated and the differences between the localization sensitivity of the old and new model are pointed out. In addition to the detection of bifurcations from an initially uniform state, the stable branch of the load-displacement diagram is followed using an incremental procedure.
\end{abstract}

\section{INTRODUCTION}

As is now widely accepted, continuum modeling of progressive cracking in quasi-brittle materials such as concrete, rock, tough ceramics, or ice requires constitutive models that exhibit strain-softening. In the context of standard local constitutive models (in which the stress-strain relationship at one point is not influenced by the evolution of stress and strain at other points), the presence of strain-softening leads to serious theoretical as well as numerical deficiencies. The governing differential equations lose ellipticity (in a static formulation) or hyperbolicity (in a dynamic formulation) and the problem ceases to be well posed. These deficiencies manifest themselves in numerical calculations by spurious mesh sensitivity - strain usually localizes into a narrow band whose width depends on the size of finite elements in the mesh and converges to zero as the mesh is refined. The corresponding load-displacement diagram always exhibits snapback for a sufficiently fine mesh and the total energy dissipated by fracture converges to zero.

To remedy the situation, a device called the localization limiter must be introduced to enforce a finite width of the localization band and a finite energy dissipation. The localization width is closely related to the so-called characteristic length of the material determined by the microstructure, e.g., by the size of inhomogeneities.

Several types of localization limiters have been proposed. A wide class

\footnotetext{
'Asst. Prof., Fac. of Civ. Engrg., Czech Tech. Univ., Thákurova 7, 16629 Prague, Czech Republic; formerly Postdoctoral Res. Fellow, Northwestern Univ., Evanston, IL 60208.

${ }^{2}$ Walter P. Murphy, Prof. of Civ. Engrg. and Mat. Sci., Northwestern Univ., Evanston, IL.

Note. Discussion open until December 1, 1994. To extend the closing date one month, a written request must be filed with the ASCE Manager of Journals. The manuscript for this paper was submitted for review and possible publication on July 28, 1993. This paper is part of the Journal of Engineering Mechanics, Vol. 120, No. 7, July, 1994. CASCE, ISSN 0733-9399/94/0007-1521/\$2.00 + \$.25 per page. Paper No. 6652 .
} 
of localization limiters is represented by the nonlocal continuum concept, which was introduced into continuum mechanics by Eringen $(1965,1966)$, Kröner (1967), Eringen and Edelen (1972), and others, and was proposed as a localization limiter by Bažant et al. (1984). An effective nonlocal damage model was developed by Pijaudier-Cabot and Bažant (1987) and Bažant and Lin (1988). It bears some resemblance to the crack band model (Bažant and $\mathrm{Oh}$ 1983) and to the mesh-dependent softening modulus of Pietruszczak and Mróz (1981). A differential form of the nonlocal concept (Bažant 1984) was exploited in various gradient-dependent models (Schreyer and Chen 1986; Lasry and Belytschko 1988; de Borst and Mühlhaus 1991). A more refined limiter of this type is the micropolar continuum (Cosserat and Cosserat 1909), which was extended to strain-softening problems by Mühlhaus and Vardoulakis (1987). A computational model for the elastoplastic Cosserat continuum was formulated by de Borst and Sluys (1991). Viscoplastic regularization (Needleman 1987) limits localization by adding rate-dependent terms to the constitutive equations.

\section{NEW APPROACH TO NONLOCAL AVERAGING}

One of the most powerful and computationally effective localization limiters is the concept of nonlocal averaging, first used in strain-softening analysis by Bažant (1984) and Bažant et al. (1984).

The original version of the nonlocal approach, which dealt with nonlocal total strain, led to certain numerical difficulties and resulted in a cumbersome imbricate structure of the finite-element approximation. A substantial increase of computational efficiency was achieved by later improvements based on the idea that only the quantities directly associated with strainsoftening (such as the damage, the damage energy release rate, or the accumulated plastic strain) should be treated as nonlocal, while the elastic part of the behavior should remain local. Nonlocal versions of several constitutive models were successfully implemented into finite-element codes and applied to a variety of problems by Bažant and Pijaudier-Cabot (1988), Bažant and Lin (1988), and Bažant and Ožbolt (1990). The nonlocal version (Bažant and Ožbolt 1990) of the microplane model (Bažant and Prat 1988) proved to be particularly efficient for the computer analysis of structures made of quasi-brittle materials such as concrete. However, it also became clear that the classical nonlocal concept based on an isotropic weight function has its limitations and does not allow formulating a model universally applicable to the same material under different loading conditions. More specifically, it turned out that the values of the characteristic length required to fit experimental data for very different test geometries are significantly different and therefore it cannot be regarded as a true material parameter. Moreover, the physical meaning of nonlocal averaging was not clear and theoretically supported, and so the nonlocal concept appeared as an artifice dictated merely by the need to regularize the governing differential equations.

To overcome these difficulties, a micromechanically based derivation of the nonlocal operator was presented by Bažant (1994b). This led to certain modifications of the original approach. Both the original and the new approaches start from the incremental form of a local constitutive law

$$
\Delta \boldsymbol{\sigma}=\mathbf{C}_{t}: \Delta \boldsymbol{\varepsilon}
$$

where $\Delta \boldsymbol{\sigma}$ and $\Delta \boldsymbol{\varepsilon}=$ increments of the stress and strain tensor; and $\mathrm{C}_{t}=$ 
fourth-rank tangential stiffness tensor of the material. The total stress increment is decomposed into the elastic and inelastic parts

$$
\Delta \boldsymbol{\sigma}=\mathbf{C}_{u}:\left(\Delta \varepsilon-\Delta \varepsilon^{\prime \prime}\right)=\mathbf{C}_{u}: \Delta \varepsilon-\Delta \mathbf{S}
$$

Here, $\mathbf{C}_{u}=$ stiffness tensor for unloading; $\Delta \varepsilon^{\prime \prime}=$ increment of the inelastic strain tensor; and $\Delta \mathbf{S}=$ increment of the local inelastic stress tensor. Eqs. (1) and (2) can be combined to yield the law relating the local inelastic stress increment to the strain increment

$$
\Delta \mathbf{S}=\left(\mathbf{C}_{u}-\mathbf{C}_{t}\right): \Delta \boldsymbol{\varepsilon}
$$

In the nonlocal formulation, the elastic stress increment remains unchanged while the inelastic stress increment $\Delta \mathbf{S}$ is replaced by its nonlocal value $\Delta \overline{\mathbf{S}}$. The constitutive law is now given by

$$
\Delta \boldsymbol{\sigma}=\mathbf{C}_{u}: \Delta \boldsymbol{\varepsilon}-\Delta \overline{\mathbf{S}}
$$

where the nonlocal inelastic stress increment is to be computed by applying a certain nonlocal operator on the local inelastic stress increment derived from the strain increment according to (3). In the previously used nonlocal formulation, this operator represents weighted averaging over a certain neighborhood

$$
\Delta \overline{\mathbf{S}}(\mathbf{x})=\int_{V} \Phi(\mathbf{x}, \boldsymbol{\xi}) \Delta \mathbf{S}(\xi) d \xi
$$

The scalar weight function $\Phi(\mathbf{x}, \boldsymbol{\xi})$ depends only on the distance $r=\| \mathbf{x}-$ $\xi \|$ between the "source point" $\xi$ and the "effect point" $\mathbf{x}$, and on a parameter called the characteristic length $l$ of the nonlocal continuum. The usual form of $\Phi(\mathbf{x}, \boldsymbol{\xi})$ has been either a Gaussian distribution function or a bell-shaped function with a compact support $\Phi(\mathbf{x}, \boldsymbol{\xi})=\Phi_{0}\left[1-(r / l)^{2}\right]^{2}(r \leq l)$, where $\Phi_{0}$ is a normalizing factor such that $2 \pi \int_{0}^{\infty} \Phi(\mathbf{x}, \boldsymbol{\xi}) r d r=1$.

Based on analysis of the equations describing the interaction among microcracks in an elastic medium, the following generalization of the nonlocal concept has been derived (Bažant 1994b)

$$
\Delta \bar{S}^{(i)}(\mathbf{x})=\int_{V} \Phi(\mathbf{x}, \boldsymbol{\xi}) \Delta S^{(i)}(\boldsymbol{\xi}) d \xi+\int_{V} \sum_{j=1}^{3} \Lambda^{(i j)}(\mathbf{x}, \boldsymbol{\xi}) \Delta \bar{S}^{(j)}(\xi) d \xi
$$

where $\Delta S^{(i)}, i=1,2,3,=$ increments of the principal inelastic stresses; and $\Lambda^{(i j)}(\mathbf{x}, \boldsymbol{\xi})=$ so-called crack influence function. Superscripts $(i j)$ at $\Lambda^{(i j)}$ indicate that the value of this function depends not only on the locations of the source point and the effect point, but also on the orientation of the principal directions at these points. Analysis of the stress fields in two- and three-dimensional infinite bodies with a single crack provides us with the asymptotic form of the crack influence function and shows that this function is decaying as $r^{-2}$ in two dimensions and $r^{-3}$ in three dimensions, in contrast to the much faster decay of the aforementioned averaging function $\Phi(\mathbf{x}, \boldsymbol{\xi})$. Furthermore, the crack influence function is not isotropic, i.e. it cannot be reduced to a function of the distance $r$ only. The behavior of $\Lambda$ for small values of $r$ is a statistical problem, cannot be easily derived, and has to be reasonably approximated. The following approximate (but asymptotically exact) form of the crack influence function for two-dimensional problems has been derived after certain simplifications (Bažant 1994b): 


$$
\Lambda(\mathbf{x}, \boldsymbol{\xi})=-\frac{k(r)}{2 l^{2}}[\cos 2 \theta+\cos 2 \psi+\cos 2(\theta+\psi)]
$$

where

$$
k(r)=\left(\frac{\kappa l r}{r^{2}+l^{2}}\right)^{2}
$$

The angles $\theta$ and $\psi$ characterize the orientations of two interacting cracks as shown in Fig. $1(a)$; and $\kappa=$ a nondimensional parameter roughly equal to the ratio of the average crack size and the characteristic length. The orientations $\theta$ and $\psi$ depend on stress state at each point. A rigorous statistical definition of function $\Lambda(\mathbf{x}, \xi)$ was given by Bažant $(1994 \mathrm{a}, \mathrm{b})$, but this form appears too complicated for the present solution.

A substantial simplification of (6) can be obtained by taking into account only the interactions between dominant cracks forming in the planes perpendicular to the maximum principal stress

$$
\Delta \bar{S}^{(1)}(\mathbf{x})=\int_{V} \Phi(\mathbf{x}, \boldsymbol{\xi}) \Delta S^{(1)}(\xi) d \xi+\int_{V} \Lambda^{(11)}(\mathbf{x}, \boldsymbol{\xi}) \Delta \bar{S}^{(1)}(\xi) d \xi
$$

The superscripts (1) and (11) will further be omitted to simplify the notation. Note that in the continuum description a crack is considered at every point. This is of course only the continuum smearing. The cracks are in reality discrete [for more detail, see Bažant (1994b)].

The effect of microdefect interactions on damage localization has also been taken into account in a different formulation (with some similarities to the present one) in the work of Okui et al. (1993).

\section{SIMPLIFIED ONE-DIMENSIONAL PROBLEM}

Behavior of the new nonlocal model in general multidimensional problems can hardly be treated analytically. It is feasible after implementation in a finite-element program (but this will be the subject of another paper by Ožbolt and Bažant). The present paper will focus on the basic properties of the new model, which must be examined first. The most basic property is one-dimensional (1D) localization of damage into a straight band, taking place inside an infinite layer of thickness $L$ [Fig. 1(b)]. For the case of a local continuum in which the localization limiter is introduced as a lower limit on the band width, as in the crack band model, this problem was treated in Bažant (1988a) and Bažant and Lin (1989) [see also Bažant and Cedolin (1991), section 13.3]. To make use of the simple expression for the crack influence function $\Lambda(\mathbf{x}, \xi)$ in two dimensions (in contrast to the much more complicated form for a $3 \mathrm{D}$ continuum), we will consider a plane stress situation-the dimension of the layer in the $z$-direction is assumed to be so small that the corresponding normal stress $\sigma_{z}$ is negligible. On the other hand, the dimension of the layer in the $y$-direction is very large and the corresponding normal strain $\varepsilon_{y}$ is negligible. The layer is loaded by enforcing a uniform displacement in the $x$-direction at one of the fixed boundaries, which causes an increase of strain $\varepsilon_{x}$ and a change of stress $\sigma_{x}$. To simplify the notation, the subscripts at $\sigma_{x}$ and $\varepsilon_{x}$ as well as at the corresponding stiffness coefficients are dropped. The stiffness coefficients $C_{u}, C_{t}$, and $C_{s}$ (to be defined later) are to be understood as the components $C_{1111}$ (or $C_{x x}$ ) 


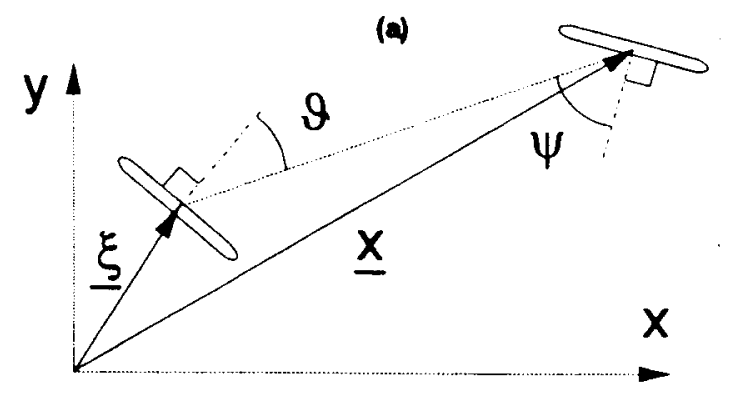

(b)

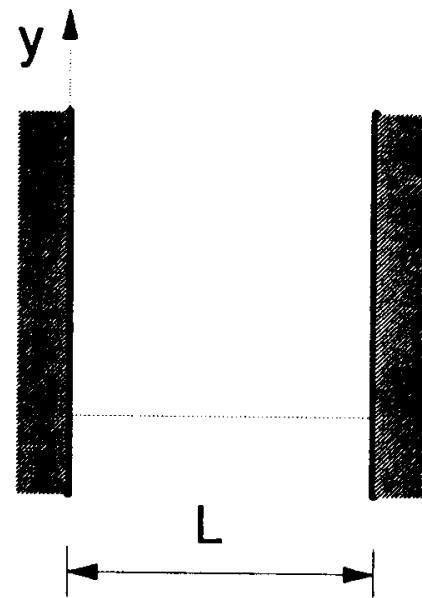

$$
y^{4}
$$
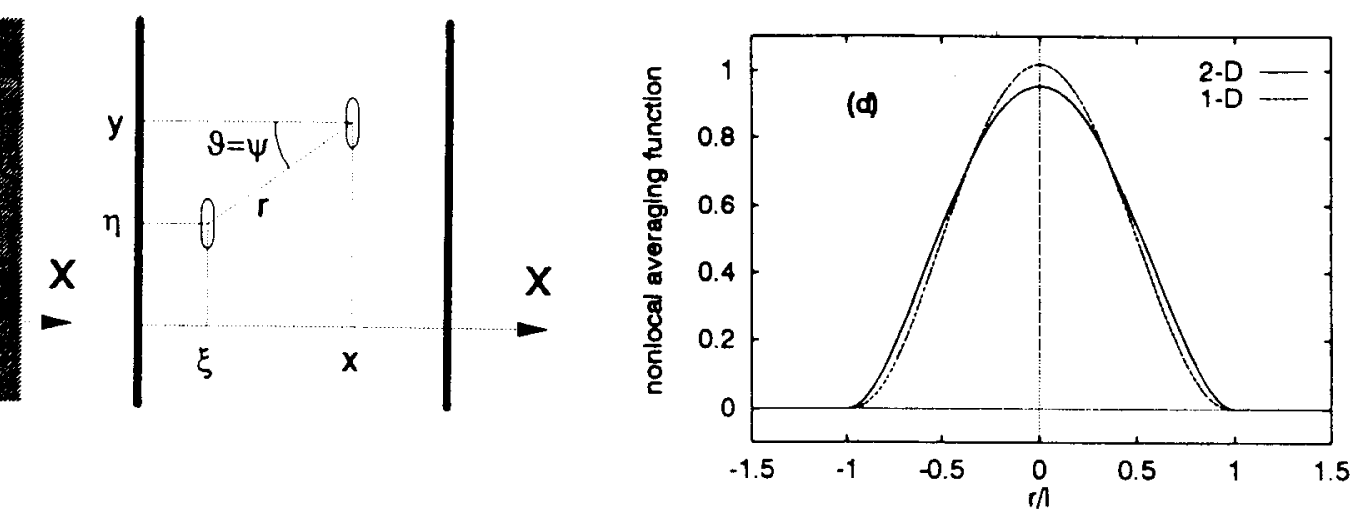

FIG. 1. (a) Orientation Angles $\theta$ and $\psi$; (b) Geometry of Infinite Layer; (c) One-Dimensional Crack Influence Function $\Lambda(r)$; (d) Nonlocal Averaging Function $\Phi(r)$

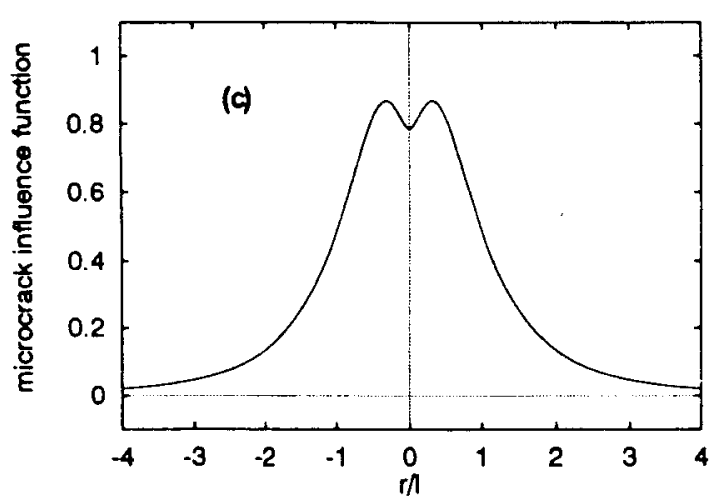

ज్ 
of the stiffness tensors $\mathbf{C}_{u}, \mathbf{C}_{t}, \mathbf{C}_{s}$, respectively, in a local constitutive law describing a plane stress problem.

Because the shear strains and stresses are zero, equilibrium in the $x$-direction requires $\sigma_{x}$ to be constant, but $\varepsilon_{x}$ can in general vary as a function of $x$. Of course, for a local continuum with a one-to-one relationship between stress and strain, $\varepsilon_{x}$ would have to be a constant, too, but the existence of a softening part in the stress-strain law can lead to strain localization and loss of uniqueness. In a local formulation, localization would occur right at peak stress and there would be no lower limit on the width of the possible localization band. As will be shown later, in the present nonlocal formulation the strain can cease to be constant even before the peak stress, but a true localization band forms only after the peak, and its width cannot decrease below a certain minimum depending on the ratio of the layer width and the characteristic length of the model as well as on the tangential modulus.

With the assumption that all the quantities depend only on one spatial variable $x$ and that the total stress (and thus also the stress increment) is independent of $x$, we can set $y=0$ in the basic equations (3), (4), and (9) and rewrite them as

$$
\begin{array}{r}
\Delta S(x)=\left[C_{u}(x)-C_{r}(x)\right] \Delta \varepsilon(x) \\
\Delta \sigma=C_{u}(x) \Delta \varepsilon(x)-\Delta \bar{S}(x) \\
\Delta \bar{S}(x)=\int_{-\infty}^{\infty} \int_{-\infty}^{\infty} \Phi(x, 0, \xi, \eta) \Delta S(\xi) d \xi d \eta \\
+\int_{-\infty}^{\infty} \int_{-\infty}^{\infty} \Lambda(x, 0, \xi, \eta) \Delta \bar{S}(\xi) d \xi d \eta \\
=\int_{-\infty}^{\infty} \hat{\Phi}(x, \xi) \Delta S(\xi) d \xi+\int_{-\infty}^{\infty} \hat{\Lambda}(x, \xi) \Delta \bar{S}(\xi) d \xi
\end{array}
$$

where

$$
\hat{\Phi}(x, \xi)=\int_{-\infty}^{\infty} \Phi(x, 0, \xi, \eta) d \eta ; \hat{\Lambda}(x, \xi)=\int_{-\infty}^{\infty} \Lambda(x, 0, \xi, \eta) d \eta
$$

The term that is additional in (9) compared to (5) should vanish in a state of uniform strain when the local and nonlocal inelastic stresses are identical. It might therefore be expected that $\hat{\Lambda} \int_{-\infty}^{x} \hat{\Lambda}(\mathbf{x}, \xi) d \xi=0$ for any value of $\mathbf{x}$. If the integral is formally transformed to polar coordinates, the integrand is given by a product of a periodic function of the angular coordinate with zero mean and another function of the radius, and on this basis the foregoing condition seems to be satisfied. A careful examination of the definition of $\Lambda(\mathbf{x}, \xi)$, however, reveals that for large values of $r, \Lambda$ behaves asymptotically as $r^{-2}$ in two dimensions and $r^{-3}$ in three dimensions, and so the integral $\int_{x}^{\infty} \int_{x}^{\infty} \Lambda(x, y, \xi, \eta) d \eta d \xi$ is not absolutely convergent. Such a problem with integrability is common to many physical problems formulated in an infinite domain and is not easy to overcome. Fortunately it disappears when dealing with real-life finite bodies or with semiinfinite bodies bounded at least in some direction. This is also the case for the layer studied in this chapter. The limits of integration in the $x$-direction are finite and, with this modification, the integral converges but is not equal to zero.

In the simple situation considered, the directions of the maximum prin- 
cipal inelastic stress at all points are aligned [Fig. 1(b)], which implies that $\theta=\psi$. With the notation $\zeta=(x-\xi) / l$, the one-dimensional crack influence function can be expressed as

$$
\hat{\Lambda}(x, \xi)=\frac{\pi \kappa^{2}}{l}\left[\frac{4 \zeta^{6}+6 \zeta^{4}+1.5 \zeta^{2}+0.25}{\left(1+\zeta^{2}\right)^{3 / 2}}-4|\zeta|^{3}\right]
$$

A surprising fact is that the resulting function is positive for all values of its arguments [Fig. 1(c)], which contradicts the intuitively expected property $\int_{-x}^{\infty} \hat{\Lambda}(x, \xi) d \xi=0$. This is a consequence of the lack of absolute integrability of the original two-dimensional crack influence function. By some tedious algebraic manipulations it can be shown that the function in the brackets in (14) decays for large $\zeta$ as $(1 / 2) \zeta^{-3}+O\left(\zeta^{-5}\right)$, and so the one-dimensional crack influence function is integrable.

\section{BIFURCATION ANALYSIS OF POSTPEAK BEHAVIOR}

\section{Formulation of Problem}

Having developed the basic framework of a one-dimensional localization problem, we now focus on the analysis of a possible bifurcation of the equilibrium path after reaching the peak stress and entering the softening regime. The local stress-strain law is assumed to be linear up to the peak, with a constant slope $C_{0}$, and then to start decaying with an initial slope $C_{s}$.

Up to the peak, the tangential stiffness $C_{r}$ and the unloading stiffness $C_{u}$ are identical and equal to $C_{0}$, and thus the local inelastic stress increments given by (10) vanish at all points of the layer. The basic equation (12) has a trivial solution $\Delta \bar{S}(x)=0$ and (11) then implies $\Delta \varepsilon(x)=\Delta \sigma / C_{0}=$ constant, which means that the strains remain uniform up to the peak. The picture dramatically changes after the peak stress is reached. A part of the layer can experience further strain increase accompanied by softening while the rest unloads in an elastic way. The unloading modulus $C_{u}$ is still equal to $C_{0}$ at all points of the layer, but the tangential modulus $C_{t}$ remains equal to $C_{0}$ only in the unloading part (denoted by $U$ ) and jumps to $C_{s}$ in the softening region (denoted by $S$ ). Eqs. (10) and (11) can be substituted into (12) to get a single integral equation for the unknown strain increment $\Delta \varepsilon(x)$

$$
\begin{aligned}
C_{0} \Delta \varepsilon(x)-\Delta \sigma=\int_{-\infty}^{\infty} \hat{\Phi}(x, \xi)\left[C_{0}\right. & \left.-C_{t}(\xi)\right] \Delta \varepsilon(\xi) d \xi \\
& +\int_{-\infty}^{\infty} \hat{\Phi}(x, \xi)\left(C_{0} \Delta \varepsilon(\xi)-\Delta \sigma\right) d \xi
\end{aligned}
$$

So far, we have kept the integration limits at minus and plus infinity. The region outside the layer can be thought of as being fixed to perfectly rigid clamps that represent a continuation of the body. So it seems to be natural to set the corresponding strain and stress increments equal to zero. Moreover, the difference $C_{0}-C_{t}(\xi)$ is zero for $\xi$ lying in the unloading region $U$ and the integrand in the first integral vanishes outside the softening region $S$. After dividing by $C_{0}$ and rearranging, (15) reads

$$
\mu \int_{S} \hat{\Phi}(x, \xi) \Delta \varepsilon(\xi) d \xi+\int_{0}^{L} \hat{\Lambda}(x, \xi) \Delta \varepsilon(\xi) d \xi-\Delta \varepsilon(x)=\frac{\Delta \sigma}{C_{0}}(\lambda(x)-1)
$$

where $\mu=1-C_{s} / C_{0} ; \lambda(x)=\int_{0}^{L} \hat{\Lambda}(x, \xi) d \xi$. The parameter $\mu$ characterizes 
the local constitutive law. It is always positive; its values between 0 and 1 indicate hardening, $\mu=1$ corresponds to a horizontal yield plateau, and $\mu>1$ to softening. On the other hand, the type of the global load-displacement diagram is determined by the sign of the stress increment $\Delta \sigma$. Naturally, $\Delta \sigma>0$ means global hardening, $\Delta \sigma<0$ global softening and $\Delta \sigma=$ 0 implies a horizontal yield plateau in the load-displacement diagram.

In addition to (16), an acceptable solution of the problem must satisfy the loading-unloading criterion

$$
\Delta \varepsilon(x) \geq 0 \text { if } x \in S ; \quad \Delta \varepsilon(x) \leq 0 \text { if } x \in U
$$

This additional condition is exploited to determine the extent of the softening and unloading regions.

When studying the bifurcation problem, the increments of stress and strain are infinitesimal and $\Delta \varepsilon(x)$ can change proportionally to $\Delta \sigma$. Because this study focuses primarily on the softening behavior, we can look for a strain increment per unit stress decrement and normalize it by the initial elastic modulus $C_{0}$ to get a nondimensional quantity $e(x)=-C_{0} \Delta \varepsilon(x) / \Delta \sigma$. In terms of this unknown function, (16) can be written as

$$
\mu \int_{S} \hat{\Phi}(x, \xi) e(\xi) d \xi+\int_{0}^{L} \hat{\Lambda}(x, \xi) e(\xi) d \xi-e(x)=1-\lambda(x)
$$

If we look for solutions that can exhibit global hardening, we can replace the definition of $e(x)$ by $e(x)=C_{0} \Delta \varepsilon(x) / \Delta \sigma$ and the right-hand side of (18) changes its sign. Finally, the case of no stress change (resulting in a plateau in the load-displacement diagram) can be treated by setting $e(x)=\Delta \varepsilon(x)$ and replacing the right-hand side of (18) by zero. The loading-unloading condition is the same for all the aforementioned cases

$$
e(x) \geq 0 \text { if } x \in S ; \quad e(x) \leq 0 \text { if } x \in U
$$

\section{DISCRETIZATION OF THE PROBLEM}

To solve (18) numerically, one can look for the values $e_{0}, e_{1}, e_{2}, \ldots$, $e_{N}$ of the unknown function $e(x)$ at a finite number of points $x_{0}=0, x_{1}$, $x_{2}, \ldots, x_{N}=L$. The unknowns can be collected to form a vector (column matrix), e. After approximating the integrals by sums, the integral (18) can be replaced by a matrix equation

$$
(\mu \mathbf{F}+\mathbf{L}-\mathbf{I}) \mathbf{e}=\mathbf{i}-\mathbf{I}
$$

where $\mathbf{F}$ and $\mathbf{L}$ are square matrices, $\mathbf{I}$ is a vector, $\mathbf{I}$ stands for the unit square matrix and $\mathbf{i}$ for the vector with all components equal to 1 . The matrix counterpart of the loading-unloading criterion (17) is then

$$
\text { Se } \geq 0 ; \quad \text { Ue } \leq \mathbf{0}
$$

where the diagonal incidence matrices $\mathbf{S}$ and $\mathbf{U}=\mathbf{I}-\mathbf{S}$ have zero and unit diagonal elements indicating whether the corresponding point lies in the softening or unloading region. To evaluate the vector $\mathbf{I}$ and the matrices $\mathbf{F}$ and $\mathbf{L}$ in (20), a numerical quadrature rule must be applied to the integrals in (18).

\section{Solution Strategies}

Because the solution of $(20)$ is subject to (21), it must be constructed in an iterative way. In a parameter study, when one wants to explore the effect 
of $\mu$ on the postpeak behavior, the softening region $S$ can be chosen in advance and one can then look for the corresponding value of $\mu$ :

1. Given $S$ and $U$, compute the matrices $\mathbf{L}$ and $\mathbf{F}$ and the vector $\mathbf{I}$.

2. Make an initial estimate of the parameter $\mu$.

3. Assemble $(\mu \mathbf{F}+\mathbf{L}-\mathbf{I})$ and $\mathbf{i}-\mathbf{I}$.

4. Solve the system of linear equations (20).

5. Check if the solution e satisfies conditions (21). If it does, output the solution and stop. If it does not, modify $\mu$ and go to step 3 .

It can be expected that softening will tend to concentrate in a band either inside the layer or at its boundary. The former situation can be denoted as U-S-U (unloading band-softening band-unloading band), the latter by S-U (softening band-unloading band). Another possibility is that all the material softens (denoted simply by $S$ ) or that there exist several separate localization bands, e.g. S-U-S or S-U-S-U.

\section{Method of Analysis and Solution for Old Nonlocal Model}

Now we need to explain how to construct an admissible solution for a given value of parameter $\mu$ and discuss the meaning of the eigenvalues and the character of the solutions for different localization modes. The computational procedure can be best illustrated by a simple example. The parameter $\kappa$ is first set to zero, which means that the two-dimensional crack influence function $\Lambda(\mathbf{x}, \boldsymbol{\xi})$ defined by (7) and (8) as well as its one-dimensional counterpart $\Lambda(x, \xi)$ defined by (14), is identically zero and the old nonlocal formulation is recovered. The matrix $\mathbf{L}$ and vector $\mathbf{l}$ then disappear from the equations and (20) takes a special, simpler form

$$
(\mu \mathbf{F}-\mathbf{I}) \mathbf{e}=\mathbf{i}
$$

Fig. 2(a) shows the strain increment profiles for an assumed localization pattern U-S-U with the total length of the layer $L=20 l$ and the assumed length of the softening region $h=10 l$. The profiles were constructed for a sequence of $\mu$ values between 0.99 and 1.03 and the loading-unloading criterion was tested for each of them. A solution satisfies the loading-unloading criterion if it is positive for $x / l \in(5,15)$ and negative everywhere else. Fig. 2(a) indicates that for $\mu=0.99$ and $\mu=1.00$, the solution is negative over the whole layer and it decreases as $\mu$ grows. Between $\mu=$ 1.00 and $\mu=1.01$, the solution jumps to large positive values and then decreases again. The interval on which it is positive shrinks and at $\mu=1.02$ it only slightly exceeds the assumed softening interval $S=(5,15)$. When $\mu$ reaches 1.03 , the interval with positive strain increments is already inside $S$. As this transition is continuous, there must be a value of $\mu$ between 1.02 and 1.03 for which the loading-unloading criterion is exactly satisfied. If a function $f(\mu)$ is defined by assigning to each $\mu$ the value of the strain increment at $x=5$, a necessary condition to satisfy the loading-unloading criterion is $f(\mu)=0$. This nonlinear equation can be solved by one of the standard techniques, e.g. by the secant method or by the Newton method. In the latter case, the derivative of $f$ is computed numerically using a difference formula. The graph of $f(\mu)$ is shown in Fig. 2(b) and it is clear that once the interval containing the root and no singularities is located, the iteration process converges without any problems.

The singular points of $f(\mu)$ correspond to the values of $\mu$ for which the coefficient matrix ( $\mu \mathbf{F}-\mathbf{I}$ ) is singular, or, equivalently, to the eigenvalues 

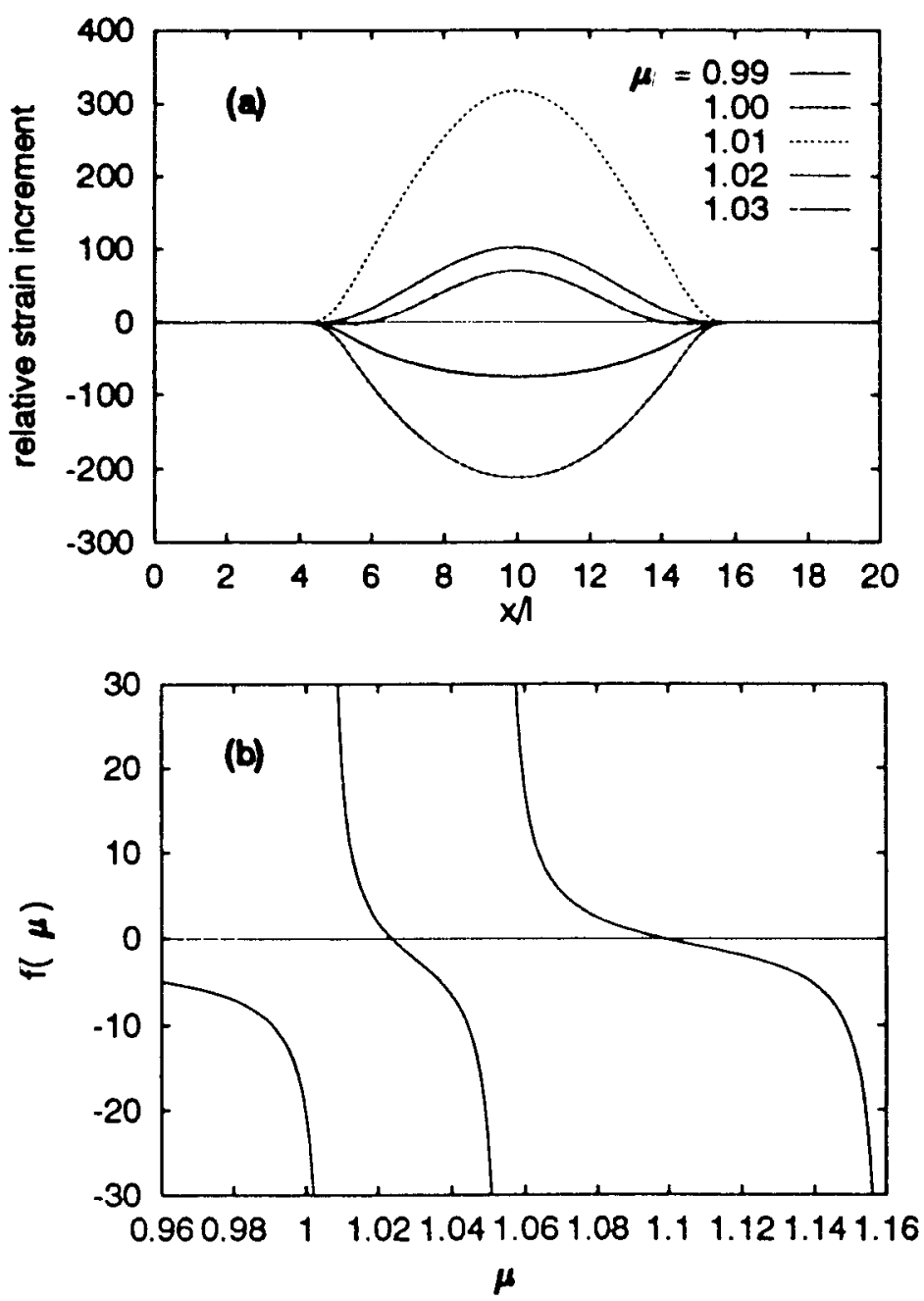

FIG. 2. (a) Solutions for Different Values of $\mu$; (b) Graph of Function $f(\mu)$

of $\mathbf{F}^{-1}$. When increasing $\mu$ from zero, the first singular value is reached approximately at $\tilde{\mu}_{1}=1.006$. At this point, the strain increments jump from large negative to large positive values [see Fig. 2(a)]. For $\mu<\tilde{\mu}_{1}$, the function $f(\mu)$ has only negative values and the corresponding solutions are not admissible. The first root of $f(\mu)$ can be found at $\mu_{1}=1.0237$ and the corresponding solution is really admissible (it satisfies the loading-unloading criterion). After the second singular value $\bar{\mu}_{2}$ is passed, another root can be detected at $\mu_{2}=1.0990$, but the corresponding solution drops below zero in a small interval in the middle of the softening band and therefore is not admissible.

The iterative procedure can be repeated for different sizes of the softening region, and each of the calculations yields one possible postpeak branch for a particular value of the parameter $\mu$. Several such solutions are depicted in Fig. 3(a). More-localized solutions require a higher value of $\mu$, i.e. a steeper slope of the descending part of the local constitutive law. Solutions with a larger softening zone are possible for smaller values of $\mu$; however, $\mu$ must always be larger than 1 , which means that the local law must exhibit softening (and not hardening or a horizontal plateau).

Other sets of solutions can be constructed for other localization patterns. 
(a)

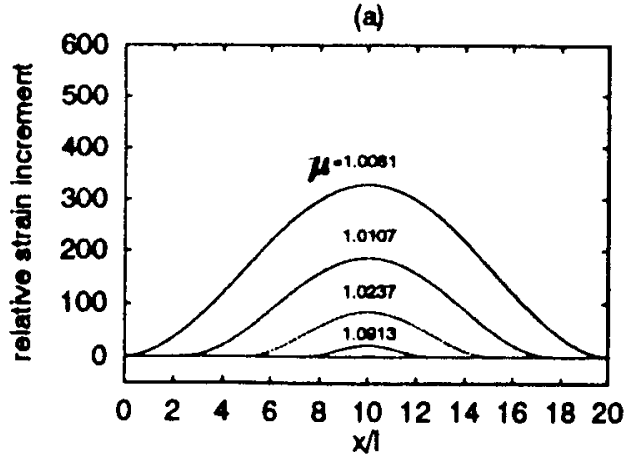

(b)

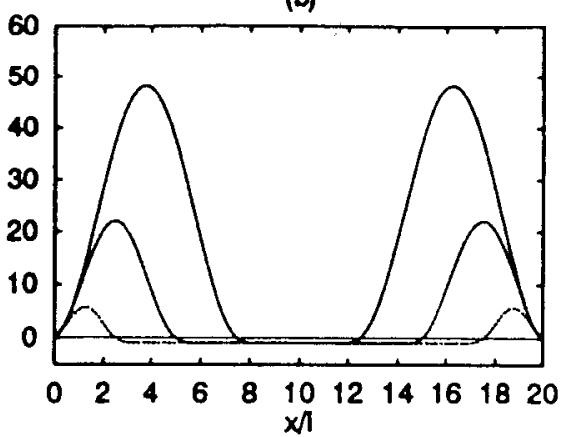

(c)

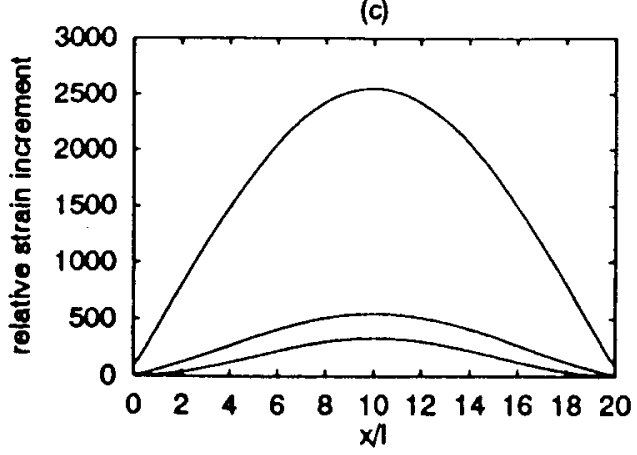

(d)
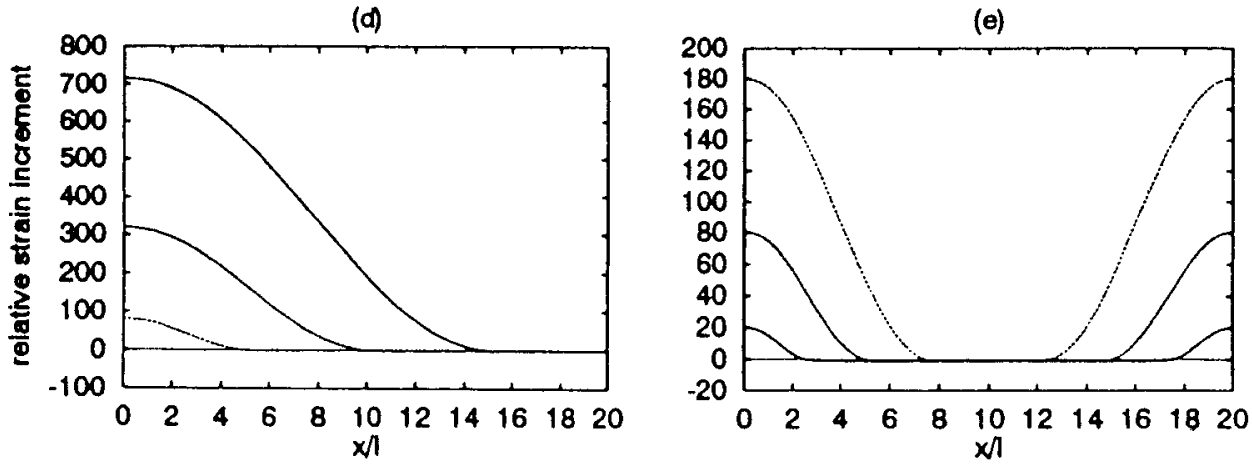

FIG. 3. Admissible Solutions: (a) U-S-U; (b) S-U-S; (c) S; (d) S-U with Renormalization; (e) S-U-S with Renormalization

It turns out, however, that, for the old nonlocal model with $\kappa=0$, the solutions are invariant with respect to a shift along the $x$-axis and therefore the $S-U$ localization profiles have the same shape as the $U-S-U$ profiles and are only shifted to the boundary. Similarly, the S-U-S profiles can be obtained by moving two identical U-S-U profiles to both boundaries [Fig. $3(b)]$. This seems to be a deficiency, because the presence of boundaries would no doubt affect the shape of the localization profiles and the corresponding values of $\mu$. The behavior of the present model can be understood if one realizes that the softening region is not affected by anything outside it, because the local inelastic stress increments in the unloading region are zero and thus they do not contribute to the nonlocal inelastic stress increments, which can be evaluated from the strain distribution in the softening zone only. That is why the position of the softening region does not make any difference and the only important thing is its length. At the same time, the strain increment profiles evaluated under the assumption of loading only 
(the $\mathrm{S}$ type of localization pattern) are highly nonuniform, with strain concentration in the middle of the layer [Fig. 3(c)]. This means that the boundaries repel strain localization.

\section{Renormalized Averaging Function}

The picture substantially changes if the averaging function $\Phi(x, \xi)$ is renormalized in the vicinity of the boundaries. The normalizing condition $\int_{-x}^{x} \Phi(x, \xi) d \xi=1$ ensures that a uniform local quantity in an infinite body leads to a uniform nonlocal quantity with the same value. If this property is to be preserved in a finite body, the integration domain must be changed to the domain of the body $V$ and, instead of the original averaging function

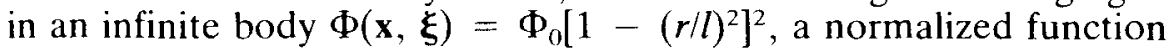

$$
\Phi_{n}(\mathbf{x}, \xi)=\frac{\Phi(\mathbf{x}, \xi)}{\int_{V} \Phi(\mathbf{x}, \xi) d \xi}
$$

must be used.

When the normalized averaging function is implemented, the model is able to exactly reproduce uniform strain increments, and also the shape of the S-U and S-U-S localization patterns becomes more reasonable [Fig. 3( $d$ and $e)]$. This formulation is therefore adopted for the subsequent development.

Due to renormalization, the matrix $\mathbf{F}$ assembled under the assumption of loading everywhere has the property that the sum of its elements in every row is equal to 1 (this is the discrete analog of the integral normalizing condition). This can be written as $\mathbf{F i}=\mathbf{i}$, where $\mathbf{i}$ is the previously defined vector with all components equal to 1 . As of course also $\mathbf{I i}=\mathbf{i},(22)$ has a solution $\mathbf{e}=\mathbf{i} /(\mu-1)$ for any $\mu \neq 1$. If $\mu>1$, this solution is admissible and it represents uniform strain increment profiles associated with stress decrements. The corresponding postpeak branch in the global load-displacement diagram has the same slope as the postpeak branch in the local constitutive law. If $\mu<1$, the solution is not admissible under the assumption that stress decreases; however, it is admissible if one assumes that stress increases. Again, the slope of the global load-displacement diagram is the same as the slope of the local constitutive law (both are positive, and hardening occurs). The transition from hardening to softening is given by $\mu=$ 1 when the coefficient matrix in (22) becomes singular. But this is perfectly consistent with the fact that the unknown function $e(x)$ defined by $e(x)=$ $-C_{0} \Delta \varepsilon(x) / \Delta \sigma$ can be introduced only if the stress change is not zero. If $\Delta \sigma$ $=0,(22)$ can be used only if $e(x)$ is replaced directly by $\Delta \varepsilon(x)$ and the right-hand side is set to zero. The homogeneous set of equations with the singular coefficient matrix F - I has infinitely many solutions, all of them multiples of the eigenvector i. The physical meaning is that for a local constitutive law with a horizontal plateau, the strain increments are uniform and arbitrarily large while the stress does not change at all.

Besides the uniform solution, several other admissible solutions may exist for the same value of the parameter $\mu$, i.e. for the same postpeak slope of the local constitutive law. It can be proven (Bažant 1988b) that the branch that will actually be followed by the real system is the one with the steepest descent. All other branches are unstable in the sense that they can never be followed spontaneously, unless an additional restriction is imposed on the system (despite the fact that the points on these branches inight be stable states). Stability of different branches of the global response existing for a 


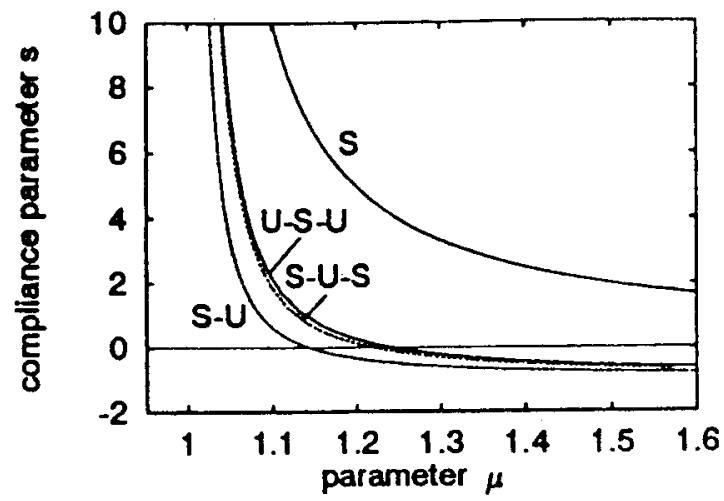

FIG. 4. Compliance Parameter for Different Localization Patterns

given local constitutive law can be evaluated by introducing a parameter related to the postpeak slope of the load-displacement diagram. To avoid difficulties with a discontinuity at snapback, the negative inverse value of the postpeak slope is used as such parameter rather than the slope itself. It is convenient to introduce a nondimensional compliance parameter

$$
s=-\frac{C_{0} \Delta \bar{\varepsilon}}{\Delta \sigma}=-\frac{C_{0}}{L \Delta \sigma} \int_{0}^{L} \Delta \varepsilon(x) d x=\frac{1}{L} \int_{0}^{L} e(x) d x
$$

whose values are positive for postpeak softening and negative if snapback occurs; $s=0$ corresponds to a vertical drop in the global load-displacement diagram indicating a loss of stability under displacement control.

It follows from the definition of the compliance parameter $s$ that the actual branch is that which minimizes $s$. To study the effect of $\mu$ on the localization pattern and on the postpeak slope, the compliance parameter was evaluated for various types of solutions and plotted against $\mu$. Fig. 4 shows such a plot for $\kappa=0 ; L=20 l$; and a renormalized averaging function $\Phi_{n}$. It is clear that the pattern S-U dominates in all situations covered by this plot. This means that the strain tends to localize into a band at one boundary (the $S-U$ pattern) rather than into a band in the middle (the U-S-U pattern) or into two symmetric bands at both boundaries (the S-U-S pattern). The decrease of $s$ with an increasing $\mu$ indicates that the postpeak slope of the load-displacement diagram is getting steeper as the slope of the local constitutive law becomes steeper. At $\mu=1.14, s$ becomes negative, which corresponds to the occurrence of a snapback. Beyond this limit, the test cannot be performed in a stable manner by controlling only the relative displacement of the boundaries.

It is interesting to check whether localization would occur for all values of $\mu>1$, i.e., whenever the local constitutive law exhibits softening. It turns out that the localization threshold lies slightly above 1 . This threshold is determined by the solution of the $S-U$ type with the maximum possible localization width $h=L=20 l$. At this extreme width, the S-U localization pattern is in fact identical with the $\mathrm{S}$ pattern denoting loading only. However, the corresponding solutions are distinct, because the S solutions are uniform while the S-U solution must satisfy the degenerated loading-unloading condition $\Delta \varepsilon(L)=0$ (the unloading region $U$ shrinks to one point). Because the matrix $\mathbf{F}$ is uniquely determined by the assumption of loading everywhere, the governing equation

$$
(\mu \mathbf{F}-\mathbf{I}) \mathbf{e}=\mathbf{i}
$$


is the same for both cases. To get two distinct solutions, the coefficient matrix $\mu \mathbf{F}$ - I must be singular and $\mu$ is therefore the reciprocal of an eigenvalue of $\mathbf{F}$. As explained before, the matrix $\mathbf{F}$ assembled under the assumption of loading only has always $\tilde{\phi}_{1}=1$ as its eigenvalue (and it turns out to be the largest eigenvalue). The corresponding $\tilde{\mu}_{1}=1 / \tilde{\phi}_{1}$ is also equal to 1 but then (25) has no solution. This means that for a local constitutive law with a horizontal plateau, the global response does not exhibit softening. The second largest eigenvalue $\tilde{\phi}_{2}$ of $\mathbf{F}$ is smaller than 1 and its inverse value $\tilde{\mu}_{2}=1 / \tilde{\phi}_{2}$ is the critical value of $\mu$ for which localization starts. Because $\operatorname{det}\left(\tilde{\mu}_{2} \mathbf{F}-\mathbf{I}\right)=0$, solutions of (25) can be written as

$$
\mathbf{e}=\frac{1}{\tilde{\mu}_{2}-1} \mathbf{i}+\alpha \tilde{\mathbf{e}}_{2}
$$

where $\tilde{\mathbf{e}}_{2}=$ eigenvector of $\mathbf{F}$ corresponding to the eigenvalue $\tilde{\phi}_{2}$; and $\alpha$ $=$ an arbitrary constant. Only solutions with all components nonnegative are admissible and the one with the last component equal to 0 is the initial solution of the S-U type. When $\mu$ is increased, the softening band $S$ ceases to extend over the whole layer and starts shrinking.

A similar analysis has been performed for other widths of the layer and the effect of the layer width $L$ as an additional parameter has been investigated. As expected, narrow layers are less suceptible to localization than wide ones, and higher values of $\mu$ are needed to produce results similar to those for wide layers. Three important characteristics of the localization sensitivity can be defined.

- $\tilde{\mu}_{1}=1 \ldots$ transition from global softenng to global hardening

- $\bar{\mu}_{2} \ldots$ onset of localization

- $\mu_{s}$... snapback, loss of stability

The values of $\tilde{\mu}_{1}$ and $\bar{\mu}_{2}$ can be determined by an eigenvalue analysis of the matrix $\mathbf{F}$, and while $\mu_{s}$ must be solved for by iteratively looking for the value of $\mu$ causing the compliance parameter $s$ to be zero. The characteristic values $\bar{\mu}_{1}, \tilde{\mu}_{2}, \mu_{s}$ are plotted against the ratio $L / l$ in Fig. 5. Depending on the layer width and the slope of the local constitutive law, four types of behavior can be distinguished, and they are graphically represented as four regions in Fig. 5.
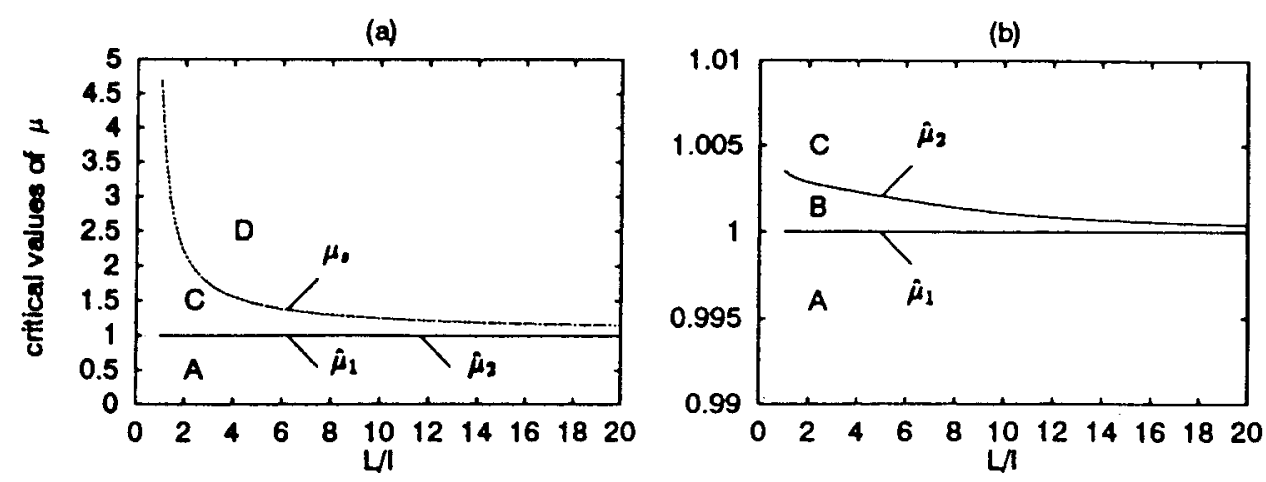

FIG. 5. Critical Values of $\mu$ Depending on Layer Width: (a) Global Picture; (b) Magnified 
- A-global hardening

- B-global softening without localization, strain increments remain uniform

- $\mathrm{C}$-localization into a softening band, stable during displacement control

- D-snapback in the global load-displacement diagram immediately after peak

\section{Analysis of New Model}

So far, all analyses and considerations have been concerned with the old localization model characterized by the absence of the additional term based on the crack influence function. How will the results be affected by the presence of this term in the integral (16) or its matrix counterpart (20)? The first striking difference is that uniform strain increments are no longer possible. This can be easily proven by substituting $\Delta \varepsilon(x)=\Delta \varepsilon=$ constant into (16), which leads to

$$
(\mu-1) \Delta \varepsilon+\frac{\Delta \sigma}{C_{0}}=\lambda(x)\left(\frac{\Delta \sigma}{C_{0}}-\Delta \varepsilon\right)
$$

Because the function $\lambda(x)$ is not constant (due to boundary effect), this equation can be satisfied only if the expression $\Delta \sigma / C_{0}-\Delta \varepsilon$ [multiplying $\lambda(x)$ on the right-hand side] is zero, but then (27) reduces to $\mu \Delta \varepsilon=0$, which can hold only if $\mu=0$ or $\Delta \varepsilon=0$. The latter case represents no change at all and can be excluded. Uniform strain increments are therefore possible only if $\mu=0$, which happens if the local constitutive law has a linear elastic part. As soon as any nonlinearity occurs strain increments become nonuniform. Fig. 6(a) shows several solutions derived under the assumption of loading only for $\kappa=0.1$ and $\mu$ ranging from 0.979 to 0.985 ; Fig. $6(b)$ shows such solutions for $\mu$ ranging from 1.002 to 1.008 .

Again, the roots of the characteristic equation

$$
\operatorname{det}(\mu \mathbf{F}+\mathbf{L}-\mathbf{I})=0
$$

mark important points where the number of admissible solutions or the character of the solution changes. The first characteristic value $\bar{\mu}_{1}$ corresponds to the transition from global hardening to global softening. Between $\tilde{\mu}_{1}$ and $\tilde{\mu}_{2}$, there is only one admissible solution for each value of $\mu$. This solution is nonuniform but all the points are softening. At $\bar{\mu}_{2}$, solutions of the S-U type start existing, and because their compliance parameter $s$ is
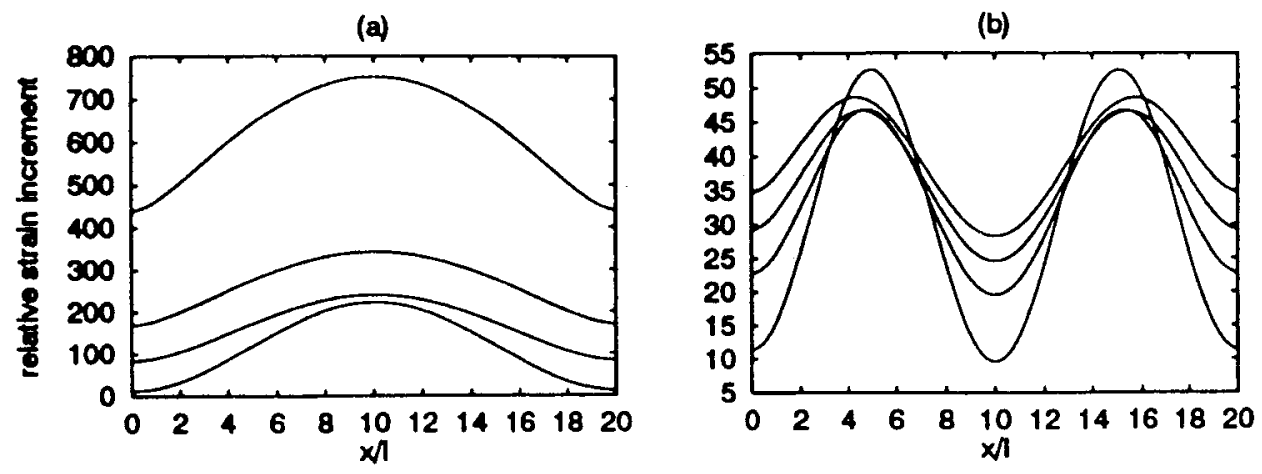

FIG. 6. Nonuniform Solutions of S Type 
(a)

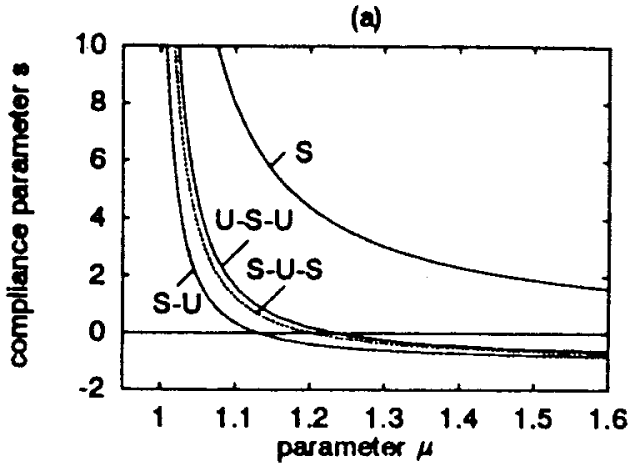

(b)

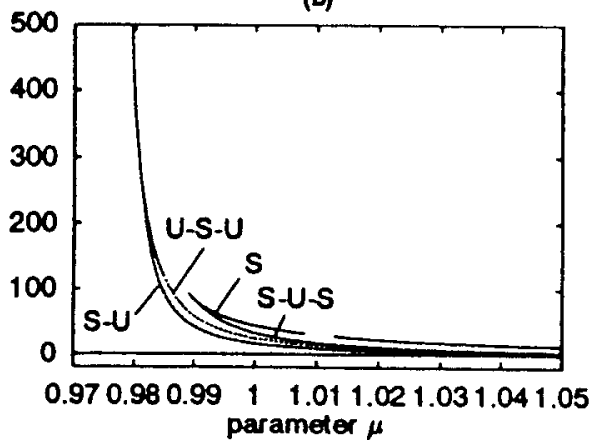

FIG. 7. Compliance Parameter for Different Localization Patterns

(a)

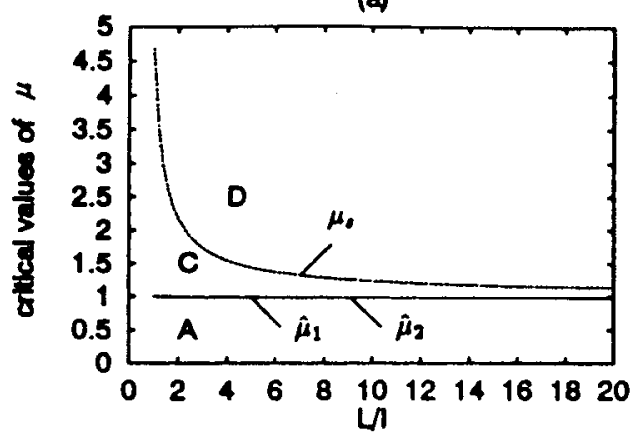

(b)

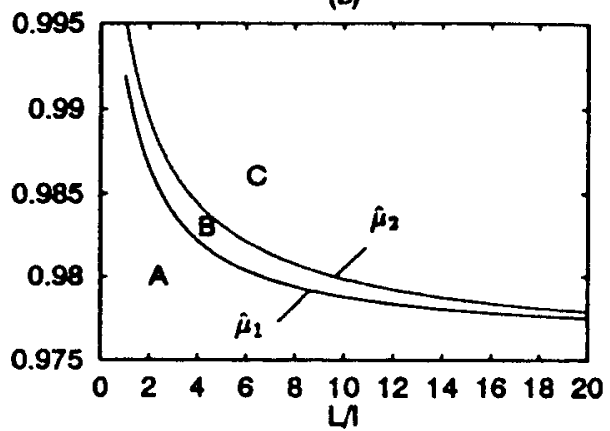

FIG. 8. Critical Values of $\mu$ Depending on Layer Width

smaller than that of the S-type solutions, the actual response follows the localized branch. In contrast to the old nonlocal model with $\kappa=0$, the $S$-type solution ceases to be admissible at some value of $\mu$ and it changes into a U-S-U solution. However, the S-type solution is "reborn" at the third characteristic value $\tilde{\mu}_{3}$ along with an S-U-S solution, and at some higher value of $\mu$ it changes its character again. The compliance parameter is plotted against the parameter $\mu$ for the most important localization patterns in Fig. 7. The figure reveals that the actual solution is of the $S-U$ type for all values of $\mu>\tilde{\mu}_{1}$. This was the case for the old nonlocal model, too, but an important difference can be noticed: The generalized nonlocal model allows strain localization even for $\mu<1$, i.e. even when the local constitutive law exhibits hardening rather than softening. The hardening slope must, however, be sufficiently small. This is demonstrated in Fig. 8 (similar to Fig. 5 for the old model), showing the four different regions as discussed at the end of the preceding subsection.

\section{INCREMENTAL ANALYSIS OF LOADING PROCESS}

\section{Formulation of Problem}

The previous section was devoted to the analysis of the initial directions of the postpeak branches, assuming a linear behavior up to the peak. Let us proceed to a more complicated problem-an incremental analysis of the entire stable postpeak branch, i.e. the branch starting with the lowest value of the compliance parameter defined previously. Recall that the basic integral (18) was derived under the assumption that the unloading modulus $C_{u}(x)$ be everywhere equal to the initial modulus $C_{0}$ and the tangential 
modulus $C_{t}(x)$ be equal to the softening modulus $C_{s}$ in the softening region $S$ and to the initial modulus $C_{0}$ in the unloading region $U$. But this is the case only in a uniform state with no damage. After a finite nonuniform increment is applied, the values of $C_{u}(x)$ and $C_{r}(x)$ in general change, except for one situation - the bilinear local constitutive law with unloading as in plasticity (by which we mean unloading with the initial slope $C_{0}$ ). In this special case, strain increments grow proportionally to the decreasing stress until the local stress drops down to zero at the first point of the body. All the other local constitutive laws require a generalization of (18).

The derivation can follow the same line as for the bifurcation analysis but the moduli must be treated as functions rather than constants. Introducing two auxiliary functions

$$
\mu(x)=\frac{C_{u}(x)-C_{t}(x)}{C_{0}} ; \quad v(x)=\frac{C_{u}(x)}{C_{0}}
$$

the integral equation governing the problem under consideration can be derived

$$
\int_{S} \hat{\Phi}(x, \xi) \mu(\xi) e(\xi) d \xi+\int_{0}^{L} \hat{\Lambda}(x, \xi) \nu(\xi) e(\xi) d \xi-v(\xi) e(x)=1-\lambda(x)
$$

The matrix counterpart of (30) can be written in a form similar to (20)

$$
\left(\mathbf{F}_{\mu}+\mathbf{L}_{v}-\mathbf{N}_{\mathbf{v}}\right) \mathbf{e}=\mathbf{i}-\mathbf{I}
$$

Subscripts $\mu$ and $\nu$ emphasize that the matrices depend on the current values of the functions $\mu(x)$ and $\nu(x)$, which are in turn determined by the total strain profile $\varepsilon(x)$, the profile of the maximum previously reached strain $\varepsilon_{\max }(x)$, and the local constitutive law. Note that (31) is a generalized form of (20) but describes the problem on a different level-as an evolution equation characterizing an entire branch of the equilibrium path rather than a bifurcation from a given state.

\section{Bilinear Local Constitutive Law}

As an example, consider a bilinear local constitutive law with damage (unloading to the origin); Fig. $9(a)$. Let $\varepsilon_{p}$ be the strain at peak stress and $\varepsilon_{f}$ the strain at complete failure. Given the current strain $\varepsilon$ and the maximum previously reached strain $\varepsilon_{\max }$, the parameters $\mu$ and $v$ at the given material point can be evaluated as follows:

1. Virgin loading $\left(\varepsilon_{\max }<\varepsilon_{p}\right)$

$$
\mu=0 ; \quad \nu=1
$$

2. Softening $\left(\varepsilon_{p} \leq \varepsilon_{\max }<\varepsilon_{f}\right)$

$$
\mu=\frac{\varepsilon_{f} \varepsilon_{p}}{\varepsilon_{f}-\varepsilon_{p}} \frac{1}{\varepsilon_{\max }} ; \quad \nu=\frac{\varepsilon_{p}}{\varepsilon_{f}-\varepsilon_{p}}\left(\frac{\varepsilon_{f}}{\varepsilon_{\max }}-1\right)
$$

3. Complete fracture $\left(\varepsilon_{f} \leq \varepsilon_{\max }\right)$

$$
\mu=0 ; \quad \nu=0
$$

Note that the unloading region is excluded from the integral containing 

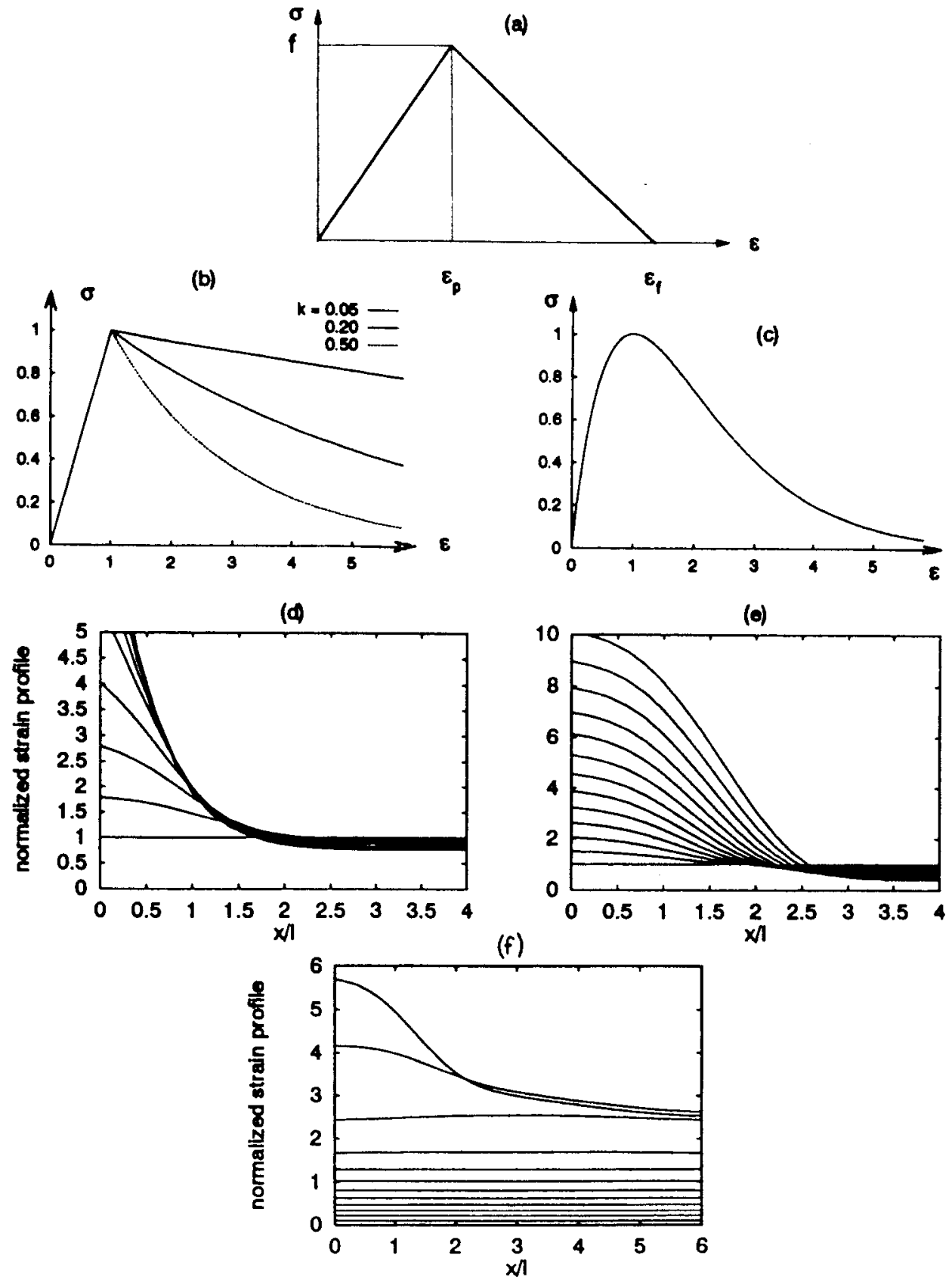

FIG. 9. (a) Bilinear Law; (b) Linear-Exponential Law; (c) Exponential Law. EvoIution of Total Strain Profile for: (d) Bilinear Law; (e) Linear-Exponential Law; $(f)$ Exponential Law

$\mu(x)$ and therefore it is not necessary to make a difference between softening and unloading-reloading after previous damage ( $v$ is the same for both cases).

Typical load-displacement diagrams start by a linear elastic part that exactly corresponds to the local constitutive law, because in the absence of inelastic stress increments, the local and nonlocal stress is the same. The load-displacement diagram bifurcates right at peak and, according to the results presented in the previous section, the actual branch is that which 
(a)

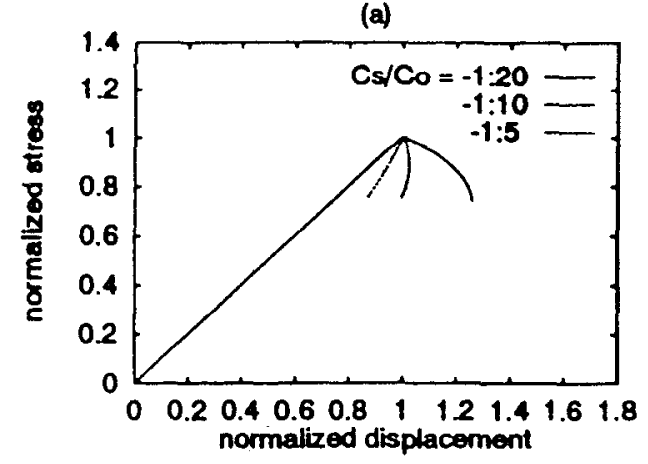

(b)

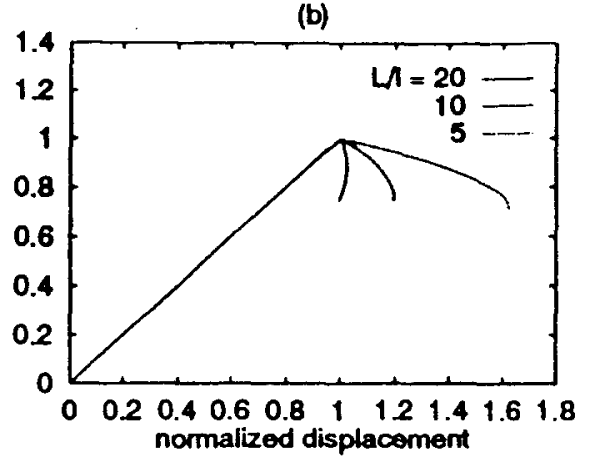

FIG. 10. Load-Displacement Diagrams: (a) for Different Local Postpeak Slopes; (b) for Different Sizes

represents localization into a softening band at one boundary. As the loading continues, the localization band becomes narrower [Fig. $9(d)$ ] and the loaddisplacement diagram becomes steeper (Fig. 10) until a snapback occurs.

The global response was followed up to the snapback or even beyond it for layers of different sizes and for local constitutive laws with different postpeak slopes. As expected, the global response is more brittle for steeper local postpeak slopes characterized by the ratio $C_{s} / C_{0}[$ Fig. $10(a)]$ and for larger relative sizes $L / l[$ Fig. $10(b)]$.

\section{Nonlinear Local Constitutive Laws}

All the load-displacement diagrams constructed in the previous subsection tend to snap back, even for small sizes and for small postpeak slopes in the local constitutive law. The reason is that, as the strain increases, the tangential modulus $C_{t}=C_{s}$ remains constant while the unloading modulus $C_{u}$ decreases to zero and so the parameter $1-C_{t} / C_{u}$ grows without any bounds. To model long tails in the load-displacement diagram with a progressively decreasing slope, the local constitutive law must exhibit a similar type of behavior. One of the simplest examples is given by a law linear up to the peak with a subsequent exponential decay [Fig. 9(d)]

$$
\sigma=f \frac{\varepsilon}{\varepsilon_{p}} \text { if } \varepsilon \leq \varepsilon_{p} ; \quad \sigma=f \exp \left[-k\left(\frac{\varepsilon}{\varepsilon_{p}}-1\right)\right] \quad \text { if } \varepsilon \geq \varepsilon_{p}
$$

where $k=$ a nondimensional constant controlling the initial postpeak slope. Large values of $k$ indicate a steep postpeak slope. Unloading is assumed to follow the initial slope. This type of a local constitutive law results in a progressive increase of the width of the localization band [Fig. $9(e)$ ] and the corresponding global load-displacement diagrams are quite reasonable [Fig. 11(a)].

As the most complex example, let us consider a case in which the local constitutive law is nonlinear even before the peak stress. A simple law of this type is given by [Fig. $9(c)$ ]

$$
\sigma=C_{0} \varepsilon \exp \left(-\frac{\varepsilon}{\varepsilon_{p}}\right)
$$

This can be again combined either with unloading to the origin (damage) or unloading with the initial slope (plasticity). The former case is studied 
(a)

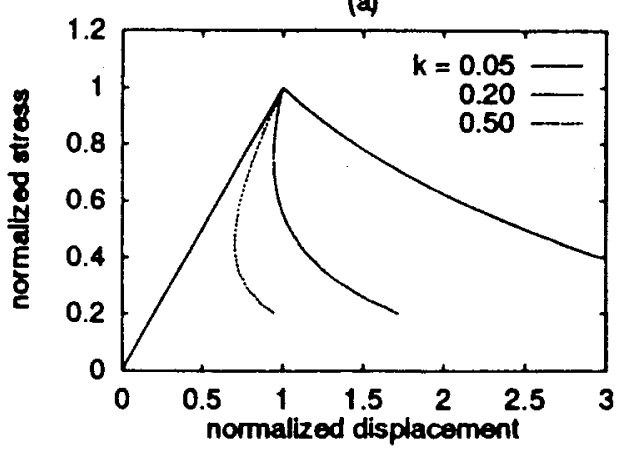

(b)

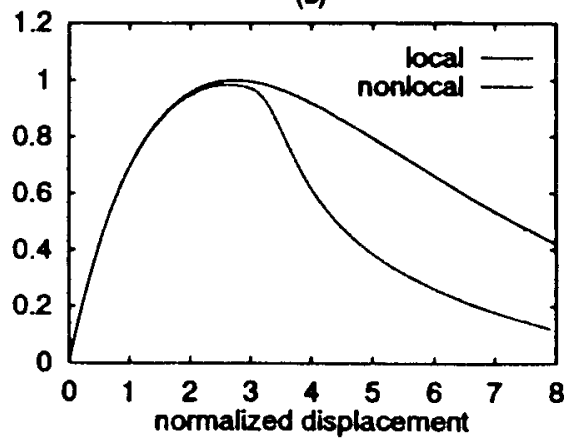

FIG. 11. Load-Displacement Dlagram: (a) Linear-Exponential Law; (b) Exponential Law

here. The incremental solution must begin with zero stress and displacement, and the normalized load-displacement diagram starts slightly deviating from the local constitutive law even in the prepeak range [Fig. 11(b)]. The evolution of the total strain profiles is depicted in Fig. 9(e) (for the law with damage). It is clear that, in the prepeak range, the strain at all the points is increasing but not uniformly. Soon after the peak stress, the solution bifurcates to a stable branch corresponding to localization in a band at one boundary. The width of the localization band then progressively increases.

\section{CONCLUSIONS}

The performance of a new nonlocal model proposed by Bažant (1994b) was tested on the problem of strain localization in a semiinfinite layer, which can be reduced to an integral equation for a single unknown function of one variable, with an additional loading-unloading condition. The following conclusions about the localization properties of the model can be drawn.

1. The conventional nonlocal model with an isotropic averaging function without renormalization cannot capture strain localization at the boundaries. Localized strain profiles are invariant with respect to a shift and not affected by the proximity of the boundary.

2. With a renormalized averaging function, the conventional nonlocal model leads to uniform strain increments in the hardening regime and in the softening regime with a very small postpeak slope. The strain increments localize into a band at one boundary if the postpeak slope of the local constitutive law exceeds a certain minimum value, which depends on the size of the layer. Large postpeak slopes of the local constitutive law result in a snapback.

3. The new nonlocal model, which contains an integral describing the effect of orientation-dependent crack interactions, leads to nonuniform strain profiles as soon as the local constitutive law deviates from linearity. The global load-displacement diagram can start softening even before the peak in the local constitutive law is reached. Similarly, the solution can bifurcate already in the (locally) hardening regime.

4. The present method of analysis was used to trace the entire loading process and study the evolution of the localized strain profiles. Several local constitutive laws leading to reasonable shapes of the load-displacement diagram were presented. 


\section{ACKNOWLEDGMENT}

Financial support under AFOSR Grant 91-0140 to Northwestern University is gratefully acknowledged. The development of the crack influence function was partially supported by the Center for Advanced Cement-Based Materials at Northwestern University.

\section{APPENDIX. REFERENCES}

Bažant, Z. P. (1984). "Imbricate continuum and its variational derivation." J. Engrg. Mech., ASCE, 110(12), 1693-1712.

Bažant, Z. P. (1988a). "Softening instability. Part I: localization into a planar band." J. Appl. Mech., 55, 523-529.

Bažant, Z. P. (1988b). "Stable states and paths of structures with plasticity or damage." J. Engrg. Mech., ASCE, 114(12), 2013-2034.

Bažant, Z. P. (1994a). "Errata."J. Engrg. Mech., ASCE, 120(6), 1401-1402.

Bažant, Z. P. (1994b). "Nonlocal damage theory based on micromechanics of crack interactions." J. Engrg. Mech., ASCE, 120(3), 593-617.

Bažant, Z. P., Belytschko, T. B., and Chang, T.-P. (1984). "Continuum model for strain softening." J. Engrg. Mech., ASCE, 110(12), 1666-1692.

Bažant, Z. P., and Cedolin, L. (1991). Stability of structures: elastic, inelastic, fracture and damage theories. Oxford University Press, New York, N.Y.

Bažant, Z. P., and Lin, F.-B. (1988). "Nonlocal yield-limit degradation." Int. J. Numerical Methods in Engrg., 26, 1805-1823.

Bažant, Z. P., and Lin, F.-B. (1989). "Stability against localization of softening into ellipsoids and bands: parameter study." Int. J. Solids and Struct., 25, 1483-1498.

Bažant, Z. P., and Oh, B.-H. (1983). "Crack band theory for fracture of concrete." Matériaux et Constructions, 16, 155-177.

Bažant, Z. P., and Ožbolt, J. (1990). "Nonlocal microplane model for fracture, damage, and size effect in structures." J. Engrg. Mech., ASCE, 116(11), 24852505.

Bažant, Z. P., and Pijaudier-Cabot, G. (1988). "Nonlocal continuum damage, localization instability and convergence." J. Appl. Mech., 55, 287-293.

Bažant, Z. P., and Prat, P. (1988a). "Microplane model for brittle plastic material. I: theory." J. Engrg. Mech., ASCE, 114, 1672-1702.

Bažant, Z. P., and Prat, P. (1988b). "Microplane model for brittle plastic material. II: verification." J. Engrg. Mech., ASCE, 114, 1672-1702.

Cosserat, E., and Cosserat, F. (1909). Théorie des corps déformables. Hermann, Paris, France (in French).

de Borst, R., and Mühlhaus, H. B. (1991). "Continuum models for discontinuous media." Symp. on Fracture Mech. of Brittle Disordered Mat., RILEM, Noordwijk, $19-21$.

de Borst, R., and Sluys, L. J. (1991). "Localization in a Cosserat continuum under static and dynamic loading conditions." Computational Methods in Appl. Mech. and Engrg., 90, 805-827.

Eringen, A. C. (1965). "Theory of micropolar continuum." Proc., 9th Midwestern Mech. Conf., University of Wisconsin, Madison, Wisc., 23-40.

Eringen, A. C. (1966). "A unified theory of thermomechanical materials." Int. J. Engrg. Sci. , 4, 179-202.

Eringen, A. C., and Edelen, D. G. B. (1972). "On nonlocal elasticity." Int. J. Engrg. and Sci., 10, 233-248.

Kröner, E. (1967). "Elasticity theory of materials with long-range cohesive forces." Int. J. of Solids and Struct., 3, 731-742.

Lasry, D., and Belytschko, T. (1988). "Localization limiters in transient problems." Int. J. of Solids and Struct., 24, 581-597.

Mühlhaus, H. B., and Vardoulakis, I. (1987). "The thickness of shear band in granular materials." Géotechnique, London, England, 37, 271-283.

Needleman, A. (1987). "Material rate dependence and mesh sensitivity in localization problems." Computational Methods in Appl. Mech. and Engrg., 67, 68-85. 
Okui, Y., Horii, H., and Akiyama, A. (1993). "A continuum theory for solids containing microdeffects.” Int. J. of Engrg. Sci., 31(5), 735-749.

Pietruszczak, S. T., and Mróz, Z. (1981). "Finite element analysis of deformation of strain-softening materials." Int. J. Numerical Methods in Engrg., 17, 327-334.

Pijaudier-Cabot, G., and Bažant, Z. P. (1987). "Nonlocal damage theory." J. Engrg. Mech., ASCE, 113(10), 1512-1533.

Schreyer, H., and Chen, Z. (1986). "One-dimensional softening with localization." J. Appl. Mech., 53, 791-797. 